\title{
Frontal Mucocele Masked as Upper Lid Abcess- A Case Report
}

\author{
Ng WL, Umi Kalthum MN ( $₫)$, Jemaima CH, Norshamsiah MD \\ Department of Ophthalmology, Faculty of Medicine, Universiti Kebangsaan Malaysia Medical Centre, \\ Jalan Yaacob Latif, Bandar Tun Razak, 56000 Cheras, Kuala Lumpur, Malaysia.
}

\begin{abstract}
Frontal mucocele is not commonly masked as upper lid abscess.A 72-year-old Chinese man with underlying hyperthyroidism complained of left upper eyelid swelling of 6 months duration. The swelling had persisted and worsen when intravenous antibiotic was changed oral type. Visual acuity on presentation was hand motion and reverse relative afferent pupillary defect was present. Because the swelling was large and resulted in mechanical ptosis and ophthalmoplegia, a CT imaging was performed, which showed huge left frontal mucocele eroding the supereromedial orbital rim. The left globe was displaced inferolaterally but there was no extension into brain parenchyma. Fundus examination showed pale optic disc with dull macula. Old laser marks were seen at peripheral fundus. Referral to ortholaryngologist was made and endoscopic sinus surgery and evacuation of mucopyocoele was done. Culture and sensitivity of the fluid showed no organism. He recovered well postoperatively with additional two weeks of antibiotics. We highlight the necessity of surgical drainage of mucocele, following a course of antibiotic.
\end{abstract}

Keywords: $\quad$ frontal sinus, mucocele, mucopyocoele, orbit, upper lid abcess

\section{Correspondence:}

Umi Kalthum Md Noh, Department of Ophthalmology, Faculty of Medicine, Universiti Kebangsaan Malaysia Medical Centre, Jalan Yaacob Latif, Bandar Tun Razak, 56000 Cheras, Kuala Lumpur, Malaysia. Tel: +603-91455981 Fax: +603-91456673 Email: umi_fizul@yahoo.com

\section{Introduction}

Paranasal mucocele is accumulation of mucoid secretion and desquamated epithelium within sinuses. It is a slowly expanding cystic lesion. When the sinus ostia is obstructed, it can lead to expanding cystic lesion. It could be infected and leads to abcess. Frontal and ethmoidal sinuses are frequently involved in this condition (1). Mucocele can occur at any age but majority of cases are diagnosed at 40-60 years and affects both males and females, equally.

\section{Case Report}

A 72-year-old Chinese man with underlying hyperthyroidism, complained of painful left upper eyelid swelling for the past 6 months. The swelling had increased rapidly in size within 3 weeks. He was initially admitted for a week-course of intravenous
Augmentin $1.2 \mathrm{mg}$ three times daily, which was transitioned into oral Augmentin $625 \mathrm{mg}$ three times daily. The upper lid swelling had improved, and he was discharged. However, after two days of oral antibiotic at home, he noticed the upper lid swelling increased again in size, similar to his first presentation. On examination, his visual acuity was hand motion on the left eye and 6/9 on the right eye, with presence of relative afferant pupillary defect (RAPD). The poor vision and positive RAPD was attributed to previous recurrent retinal detachment and multiple unsuccessful vitreoretinal intervention. The left swollen upper eyelid covered almost two- thirds of the cornea. It was tender and erythematous, (Fig. 1, 2) but no oozing pus was noted. The extraocular movement was mechanically limited on all levoelevation. Band keratopathy was also noted at the lower half of cornea. Otherwise, there was no anterior chamber reaction and conjunctiva was fairly white. His right eye retinal 

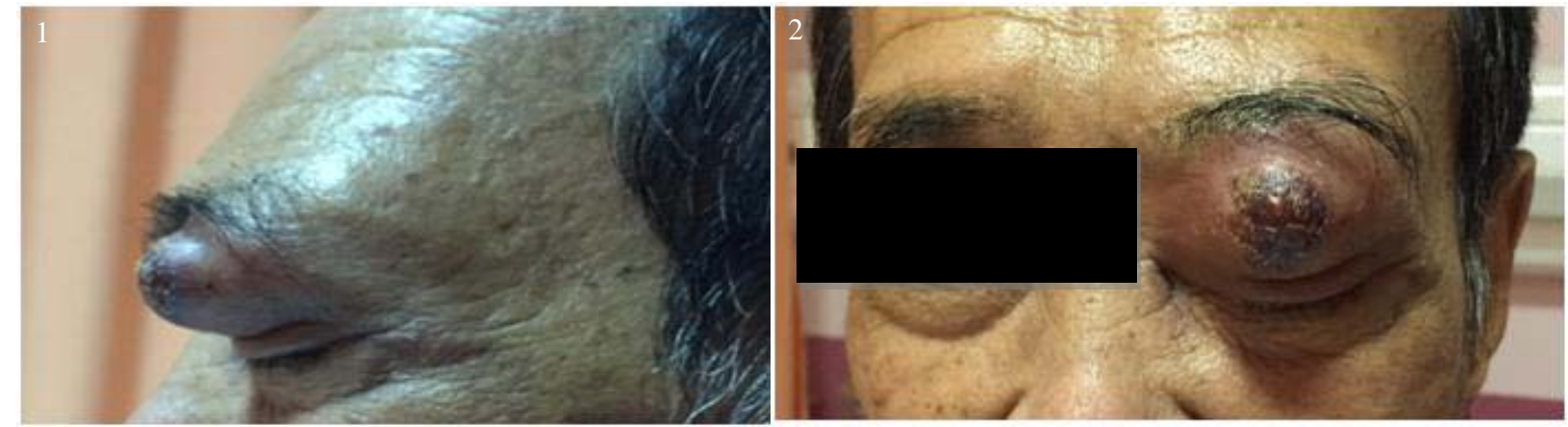

Figure 1and 2: Severe left upper lid swelling with mechanical complete ptosis and preseptal cellulitis
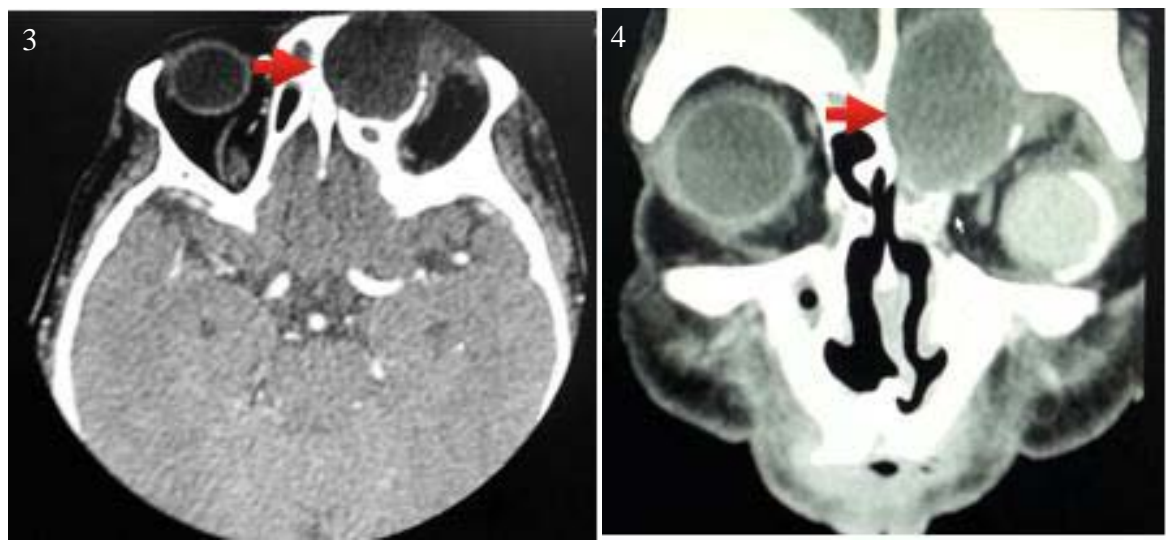

Figure 3 and 4: CT scan showing huge homogenous hypodense mass at frontal sinus extending into ethmoidal sinus and inferotemporal displacement of globe.

examination corresponded with previously repaired retinal detachment. The optic disc appeared to be pale and macula was dull. Laser marks were seen in peripheral retina.

A hypodense lesion with thin peripheral enhancement occupying the left frontal sinus, extending into ethmoidal sinus was apparent on computed tomography scan. The superomedial bony orbital rim was eroded, with inferomedial displacement of the left globe (Fig. 3,4). Fortunately, the brain parenchyma was intact.

He underwent endoscopic sinus surgery and evacuation of mucopyocoele by the ortholaryngologist, which drained greenish fluid. However, the culture and sensitivity did not yield any positive result. He was discharged with two weeks course of antibiotics, following completion on 2 days of intravenous antibiotic. During subsequent follow up his eye swelling subsided, and he remained stable.

\section{Discussion}

Mucocele of paranasal sinus is a benign lesion which consists of accumulation of mucoid secretion and desquamated epithelium within the sinus. It is expansile and may cause geographical damage to neighbouring structures including the intracranial tissue and orbit (2). It is most likely due to obstruction of the drainage ostium of paranasal sinuses and impaired sinus drainage (3). The causes of obstruction could be due to inflammation, trauma or facial surgery, mass and radiotherapy. When the accumulated mucoid secretion is infected, it may lead to abcess or pyocoele. In the present case, the swelling was clinically suggestive of infection in view of inflammatory skin changes and draining of greenish fluid intraoperatively. Secondary preseptal cellulitis due to underlying infection in the mucocele may add confusion to the clinical picture initially. Infected mucocele may also present as severe swelling and orbital cellulitis. We highlight the importance of recognizing this condition and for clinicians to abstain from doing emergent simple incision and drainage of the abcess.

The clinical presentation of mucocele varies according to the structures affected. Patients may come with headache, painful eyelid swelling, reduced visual acuity, diplopia, facial pain and nasal obstruction $(2,3,4$,$) . The floor of frontal sinus also$ makes up the superior orbital wall, hence a large enough mass within the frontal sinus results in 
downward displacement of orbit as early sign (2). Intracranial extension results in development of meningitis or cerebrospinal fluid fistula (5), with detrimental consequences and eventual mortality if left untreated.

The common differential diagnoses for the eyelid swelling include eyelid abscess, orbital cellulitis, cholesterol granuloma, encephalocele, epidermoid cyst, meningioma, neurofibroma and malignant tumours (6). We highlight a case of frontal mucocele which initially responded to intravenous antibiotic, but came back after transitions to oral regime. The possibility of poor response to antibiotics initially could be due to partially treated infection. The closed compartment of a sinus may result in granuloma formation, further isolating the infection and pus collection within. Intravenous antibiotic prevents further progression of infection, but surgical intervention is necessary to drain isolated collection. Hence, we recommend surgical debridement of pus in such cases, following initiation of antibiotic and further prophylactic antibiotic for another week onwards. Alternatively, it may be prudent to repeat imaging after 1 week of antibiotic therapy to ensure the collection is minimal and subsequent transition to oral therapy would be effective.

Imaging is essential to differentiate tumours from inflammatory causes. Although magnetic resonance is the most ideal for better soft tissue resolution, computed tomography is more widely available, and still has values in early evaluation. Furthermore, bony erosion is better seen in CT (7), which normally showed opacified sinus with thinned-out margin. MRI is only pursued when there is suspicion of soft tissue mass or neoplasm (7), and to rule out infective extension into brain parenchyme.

Small mucocele may be amenable to intravenous antibiotic therapy, especially if caught early. The treatment of large mucocele is surgical drainage of collection to allow ventilation of involved sinus. It can be achieved either externally or through endoscopic approach, with or without sinus obliteration (8). The current preferred surgical modality is via endoscopic route which is less invasive and cause less morbidity. In recurrent cases of mucocele, complete mucosal lining removal and fat obliteration of the sinus has been successfully performed (7). In this case, sinus obliteration was not necessary as he responded well to the drainage alone. Adjuvant antibiotics are indicated in the presence of infection, or more often as prophylaxis following surgical intervention.

\section{Conclusion}

Mucocele is a benign condition but can involve orbital and intracranial structures. To prevent the undetected extension, it should be diagnosed early with the aid of imaging and to be treated early. We must be cautious in making a diagnosis of a simple lid abscess, which may mask a frontal mucocele. Radiological imaging is vital to aid the diagnosis and planning the surgical approach. Proximity of mucocele to the brain may cause significant morbidity and potential mortality.

\section{References}

1. Tan CS1, Yong VK, Yip LW, Amrith S. An unusual presentation of giant frontal sinus mucocele manifesting with a subcutaneous forehead mass. Ann Acad Med Singapore 2005; 34(5): 397-8.

2. Hurley DB, Javer AR, Kuhn FA, Citardi MJ. The endoscopic management of chronic frontal sinusitis associated with frontal sinus posterior table erosion. Am J Rhinol 2000; 14(2): 113-20.

3. Chiarini L, Nocini PF, Bedogni A, Consolo U, Giannetti L, Merli GA. Intracranial spread of a giant frontal mucocele: case report. $\mathrm{Br} \mathrm{J}$ Oral Maxillofac Surg 2000; 38(6): 637-40.

4. Lund VJ, Rolfe ME. Ophthalmic considerations in fronto-ethmoidal mucoceles. J Laryngol Otol 1989; 103(7): 667-9.

5. Voegels RL, Balbani AP, Santos Junior RC, Butugan O. Frontoethmoidal mucocele with intracranial extension: a case report. Ear Nose Throat J 1998; 77(2): 117-20.

6. Benninger MS, Marks S. The endoscopic management of sphenoid and ethmoid mucoceles with orbital and intranasal extension. Rhinology 1995; 33(3): 157-61.

7. Aggarwal SK, Bhavana K, Keshri A, Kumar R, Srivastava A. Frontal sinus mucocele with orbital complications: Management by varied surgical approaches. Asian J Neurosurg 2012; 7(3): 135-40.

8. Galiè M, Mandrioli S, Tieghi R, Clauser L. Giant mucocele of the frontal sinus. J Craniofac Surg 2005; 16(5): 933-5. 